\title{
A Reanalysis of the Two Swimmers Problem, as Frequent Model of Michelson's Interferometric Experiment Demonstrating that Transversal Path Is Not an Isosceles but a Right Triangle and the Race Will End in a Tie
}

\author{
Ioan Has ${ }^{1}$, Simona Miclaus ${ }^{1}$, Aurelian Has ${ }^{2}$ \\ ${ }^{1}$ Land Forces Academy, Sibiu, Rm. Valcea, Romania \\ ${ }^{2}$ Independent Researcher, Rm. Valcea, Romania \\ Email: hasavo@yahoo.com,simo.miclaus@gmail.com, hasaurelian@yahoo.com
}

How to cite this paper: Has, I., Miclaus, S. and Has, A. (2018) A Reanalysis of the Two Swimmers Problem, as Frequent Model of Michelson's Interferometric Experiment Demonstrating that Transversal Path Is Not an Isosceles but a Right Triangle and the Race Will End in a Tie. Journal of Applied Mathematics and Physics, 6, 1507-1521. https://doi.org/10.4236/jamp.2018.67127

Received: June 26, 2018

Accepted: July 24, 2018

Published: July 27, 2018

Copyright $\odot 2018$ by authors and Scientific Research Publishing Inc. This work is licensed under the Creative Commons Attribution International License (CC BY 4.0).

http://creativecommons.org/licenses/by/4.0/

\begin{abstract}
The article initially reviews various works describing the physical model (PM) of Michelson's interferometric experiment (ME), represented by the race between two swimmers Sw1, Sw2 (or boats, or planes, or sound signals, etc.). The two swimmers must each swim the same distance, but Sw1 will swim along the river flow, and Sw2 will swim perpendicularly to this direction. In all such works, it is considered that Sw2's path will require less time and that it will reach the start point first. However, in this work, it has been determined that in order to make this possible, Sw2 must not observe the orthogonality rule of his start direction. This action would be deceitful to the arbiters and thus considered as non-fair-play towards Sw1. The article proves by swimming times calculus, that if the fair-play rules are observed, then the correct crosswise path (in water reference frame) is a right triangle instead of the isosceles triangle considered by Michelson. Consequently, the two times shall be perfectly equal and the race ends in a tie, and the myth of Sw2 as the race winner shall be debunked. Note that the same result shall also be applicable to Michelson's interferometric experiment (ME) as well as to any similar experiment. Therefore, utilising the isosceles triangle as the transversal path in $\mathrm{PM}$ and also in ME is an erroneous act.
\end{abstract}

\section{Keywords}

Michelson Experiment, Two Swimmers Model, Swimming Times Calculation, Right Triangle Correct Transversal Path, Error of Isosceles Triangle for 


\section{General Presentation of Physical Models of Michelson's Experiment}

Many works discuss Michelson's interferometric experiment (ME) in terms of a mechanical or physical model (PM). A PM serves as an analogue to the ME experiment, which is presented as a (hypothetical) race between two swimmers, Sw1 and Sw2 (alternatively, two boats, two airplanes, two sound signals, etc.). Both swimmers swim with the same constant speed, $c$ and same distance, $l$. The swimmers make the distance 1 , and return to the starting point. The race takes place on a river that flows with speed $v$. However, the swimmers swim at different directions on the river. The first swimmer Sw1 takes the path or the route $l_{1}=l_{1}^{\prime}+l_{1}^{\prime \prime}$, where the distance $l_{1}^{\prime}$ is oriented right (opposite) of the river flow direction, $v$. The second swimmer, Sw2, will take the path $l_{2}=l_{2}^{\prime}+l_{2}^{\prime \prime}$; the initial distance $l_{2}^{\prime}=l_{1}^{\prime}$, and is perpendicular to the river flow direction $v$ and thus also perpendicular to direction $l_{1}^{\prime}$.

This orthogonality condition of the two initial paths, $l_{2}^{\prime}$ and $l_{1}^{\prime}$ is mandatory, in order for the two experiments, ME and PM, to be similar. In the ME setup, the two initial light beams, 1' and 2', start at orthogonal directions. The beams head toward a semi-transparent splitter $\mathrm{P}$, from which beams 1' and 2' egress. Plate $P$ is $45^{\circ}$ sloped against the incident rays. Direct beam, $2^{\prime}$ permeates the splitter while beam 1' is reflected by the splitter. The beams then form between them a $90^{\circ}$ angle, according to the reflection laws of the geometrical optics.

However, it must also be mentioned that none of the physical (in fact mechanical) models (PMs) from the physics literature clearly and precisely establish the reference frame $(\mathrm{RF})$ for the displacements and movements. The chosen $\mathrm{RF}$ also essentially depends the accuracy of the analysis itself. This entanglement occurs because at least two local reference frames RF may be considered: the block-start or bank attached frame (BF) (the equivalent of ME interferometric device) or the water-attached frame (WF) (the equivalent of ME ether).

And for the accurate analysis of any model PM, one should specify from the beginning what is the adopted reference frame, RF. In our analysis, we will observe this rule in each and every figure or description.

However, here it must also be mentioned that in any and in all descriptions of the PMs encountered in the physics literature, one "demonstrates" that the time of swimming the transverse route is shorter than the longitudinal one, and hence Sw2 wins the race. This conclusion has become a myth for many young generations of high school or college graduates.

In the next sections, we will demonstrate that if the race would run "fair-play", both swimmers Sw1 and Sw2 would reach the start point at the exact same time. 
This myth would require a correction in this regard, i.e., "the race ends in a tie".

\section{Description of Some Physical Models from Literature}

We will hereinafter describe details and observations regarding various presentations of the speciality literature for the physical model PM with the two swimmers, which is considered by their authors similar to the Michelson's interferometric experiment ME.

We have selected from the usual physics literature of average level, few such examples, from the Internet and from works addressed to young people. Young people could be deeply influenced by such examples (as were these authors) that if proven wrong, may have a negative impact with more profound consequences on future physicists.

1) Thus in [1] Jurgen Freund states (referring to a boat, comparable with the swimmer): "When the boat is sailing at right angles to the banks it has to turn its bow slightly upstream and thus reaches the velocity $\sqrt{\left(c^{2}-v^{2}\right)}$ relative to the banks".

But we observe that the mention turn its bow slightly upstream indicates a clear lack of orthogonality in start direction of second boat.

2) In [2] David Harrison says (referring to a raft and two markers, comparable with two banks): "Now the raft and markers are being towed to the left. In this case, the race will no longer be a tie. In fact is not too hard to show that swimmer 2 wins this race".

In an Annex the same transversal velocity $\sqrt{\left(c^{2}-v^{2}\right)}$ is as in 1$)$. above, which indicate a clear lack of orthogonality in the starting direction of second swimmer Sw2, including in an animated presentation of the race.

3) Austin Gleeson [3] says: “A stream of width $D$, is flowing with the speed $v_{0}$ from left to right. A swimmer whose speed is in still water $v$ wants to swim across and back reaching the other bank at a point opposite the starting point. The resultant velocity which is directed across the creek is thus $\sqrt{\left(c^{2}-v^{2}\right)}$ ".

No mention is made about the orthogonality of $d_{1}^{\prime}$ and $d_{2}^{\prime}$ paths for which, this condition is clearly absent.

4) However, Michael Fowler [4] says: "The swimmer going across the flow is trickier. It won't do simply to aim directly for the opposite bank, the flow will carry the swimmer downstream (Figure 1).

To succeed in going directly across, the swimmer must actually aim upstream at the correct angle".

Here we observe a clear mention about the lack of orthogonality of paths $d_{1}^{\prime}$ and $d_{2}^{\prime}$ from the text mentioning at the correct angle but Fowler associates the lack of orthogonality with a trick.

5) Although in [5] Bernard Jaffe does not specify the conditions in which "boater 2 crosses the water flow" but he chooses for the boater a path after the hypotenuse of the right triangle of the speeds $v$ and $c$. The figure attached to the text shows that boater 2 runs the $27.5 \mathrm{~m}$ of the river width after the hypotenuse. 


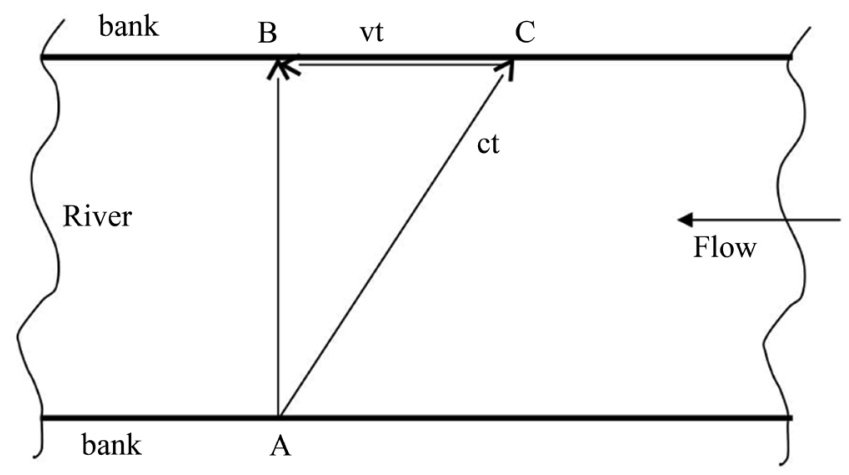

Figure 1: In time $t$ the swimmer has moved $c t$ relative to the water and been carried downstream a distance $v t$

The swimmer going across the flow is trickier. It won't do simply to aim directly for the opposite bank-the flow will carry the swimmer downstream. To succeed in going directly across, the swimmer must actually aim upstream at the correct angle (of course a real swimmer would do this automatically). Thus, the swimmer is going at 5 feet per second. at an angle, relative to the river. and being carried downstream at a rate of 3 feet per second. If the angle is correctly chosen so that net movement is directly across, in one second the swimmer must have moved four feet across. The distance covered in one second will form a 3, 4, 5 triangle. So at a crossing rate of 4 feet per second, the swimmer get across in 25 seconds and back in the same time, so a total of 50 seconds The cross-swimmer wins. (on page 13 for the swimmer going parallel to river flow resulted 62,5 seconds).

Figure 1. The model PM with the swimmer Sw2 as trickier (Figure 1 and text, extracted from [4]).

So his mention "perpendicularly to the water flow..." is clear untrue due to hypotenuse path, and without specifying the reference frame considered there by the author.

6) However in [6] George Gamow directly specifies: "In this case, the delay is due (on the transversal route of the river run by a motorboat) to the fact that the boat, in order to go from boat bridge 1 to boat bridge 3 , it must advance slightly sidewise to compensate the current drift".

Thus, Gamow admits the path 2' shows a sidewise advance and this is an acknowledgement that path 2' is not orthogonal to path 1'.

7) In [7] D. Ciubotaru e. a. says: "The boat's movement speed (from which sound signals with sound speed $v_{\mathrm{s}}$ are emitted) may also be determined by having a reflecting area $R_{2}$, in such a way that the arm $S R_{2}=I_{2}$ is perpendicular to the boat's movement direction. From Figure 1.3 from [7], one may determine the time $\tau_{2}$ in which the sound covers the distance $S^{\prime} R_{2}+R_{2} S \ldots \tau_{2}=2 l_{2} / \sqrt{\left(v_{s}^{2}-v^{2}\right)}$ ".

But in Figure 1.3 from [7], the path of the sound is again an isosceles triangle, which indicates a clear lack of orthogonality in the starting direction of the second sound signal (Sw2).

8) Finally here, according to Thomas D. Le [8]: “To compensate, Boat 2 has to head into the current (upstream from the work) at a certain angle so that the combined effect of $c$ and $v$ would allow it to reach Pier B". And in Figure from page 23 of [8], the path of the boat is the hypotenuse of the right triangle, which indicates a clear lack of orthogonality in the starting direction of Boat 2 (Sw2).

And such analyses of PM model in which the lack of orthogonality in the 
starting direction of second swimmer Sw2 may comprise all published works on the above subject about PM models, known by us.

\section{Findings and Observations on Previous Physical Models}

As we further advance, we will show important details and observations regarding some of the physical models PMs previously described in Sec. 2.

We note here that in every previous description of the PM analogues for the ME, the respective authors' works of description and analysis of the two paths, $I_{1}$ and $l_{2}$ contain an error in which the complete similarity between ME and PM is disregarded.

The disregarded similarity refers to how the direction of initial path $l_{2}$ was selected within the PM. We observe that $l_{2}$ of swimmer Sw2 is allowed to deviate from the orthogonal direction to the water speed direction $v$, because Sw2 starts at a small angle $\beta$ against the exact orthogonal direction. Such a path deviation constitutes a trick and a non-fair play towards Sw1.

Note that $\beta$ is not explicitly stated before swimmer Sw2 starts racing. It is only indirectly mentioned (a trick) because it is said that Sw2 should aim at a point $\mathrm{C}$ from the opposite bank, but situated outside the orthogonal direction.

But this inclined aiming of initial path $l_{2}$ is impossible within the conditions of the interferometric experiment (ME), in which the opposite M2 mirror does not include reference marks for inclined orientation of the light beam, which does not have the sentience in order to consent to a trick and thereby it starts the path $l_{2}^{\prime}$ exactly at $90^{\circ}$.

And in the PM model of ME, no personnel, and no orientation sign for Sw2 is found on the opposite bank that can indicate him the inclination angle $\beta$.

Thus, the PM serving as an analogue of ME may only succeed in practice as Michelson and authors (mentioned in Sec. 2) proposed, only if somehow Sw2 can benefit from the indication of the inclination angle $\beta$, exactly at the start-up moment. Nevertheless, this would be the case of a trick towards Sw1.

If the PM would be deployed in the conditions of an official race or even of an Olympic contest, the organizers would not allow such deviation from the orthogonality rule of paths $I_{1}$ and $l_{2}$ offering to inform Sw2 the angle $\beta$ before starting.

This means that swimmer Sw2 assigned on path $l_{2}$ should also start orthogonally on block start, thus perpendicular to path $l_{1}$ including perpendicular to the water speed direction $v$. This situation obviously is possible, only without indicating to Sw2 an inclined/shifted mark from the opposite bank and without informing him the angle $\beta$. Additionally, the angle $\beta$ also depends on the river flow speed, $v$, which should not be known by any of the contestants.

We notice that Michael Fowler roughly characterizes the swimmer Sw2 as a trickster, and we must agree that M. Fowler is perfectly right. This is because swimmer Sw2 benefits from this information, which is not allowed in a fair play contest. This means that Sw2 benefits to win from indirect knowledge of the 
river speed $v$, and with permission to start at a non-orthogonal direction to $V$, which is non-orthogonal to the swim direction of contestant Sw1.

The basic rule in such a contest as understood by laymen is that both swimmers must start from the block start on orthogonal directions between them, just as light beams start from the semi-transparent splitter in ME. Therefore, the correct path in the PM analogue of the ME must take this rule of path-orthogonality into account. In this case, in the WF frame the correct complete crosswise path of Sw2 is a right triangle instead of isosceles triangle as demonstrated in Sec. 5.

Here we define such deviation of $l_{2}^{\prime}$ for Sw2 from orthogonal directions an essential error in the involved analyses.

Fowler's characterization of swimmer Sw2 as a trickster is perfectly applicable to all the other Sw2 contestants/swimmers in the aforementioned examples and in other works describing PM analogues for ME (including the boaters, airplane pilots, etc.).

We must emphasize that the above-indicated error in the PM, was also taken over, by Michelson in the analysis of his ME experiment [9] [10] [11]. But here we will limit ourselves to the correct analysis of the physical model PM only.

\section{Correct Reanalysis of the Two Swimmers in the Physical Model}

This reanalysis shall refer to the PM analogue on the ME, model that shall consist, for simplification, of the race between the two swimmers, Sw1 and Sw2.

The following analysis is based on the important observation in Sec. 3., where the start-up mode of Sw2 must observe the rule of fair play. This means that Sw2 should not benefit before start-up of the prior knowledge of angle $\beta$ or river speed $v$, nor should he apply $\beta$ to his start direction $l_{2}$.

Sw2 should start according to the inferred and unanimously accepted rule by the audience in such contests. The two swimmers should start from their block starts at two orthogonal directions between them.

However, an aspect that should also be specified here is very important to any cinematic analysis: the reference frame (RF) of the space, to which the movements of the relevant bodies or objects are referred to, must be clearly indicated. This means that the object or the body that the RF is attached to should be clearly indicated. In case of the PM of the two swimmers, the most useful reference frames shall be the water-attached frame (moving with the speed $v$ against the bank) WF, and the block start or bank-attached frame, BF.

One may notice that all of the previous analyses (considering PMs or ME), do not approach this aspect and thus do not specify clearly the RF adopted, nor include the reference frame WF or BF in the figures of the relevant texts, by the respective authors. For this reason, when both reference frames WF and BF are involved, misunderstandings and errors in the analysis of the phenomena may ensue, as the error of the start direction of Sw2 has systematically slipped in.

In this analysis, we will indicate which RF is adopted and even clearly mark it 
in the attached figures for each every analysis. They will be indicated by the related $O x$ and $O y$ coordinates.

We would also like to mention here that for the reference frame of time $t$, RFT, the unique frame with one uniform flow speed of the unique time $t$, according to classical physics, shall be adopted in all our analyses. The reference moment as $t=0$, could be taken at either moment of the time $t$ of the experiment, provided to have fulfilled the above condition of uniform time $t$ flow.

\section{Correct Reanalysis of the Physical Model PM of the Two Swimmers in the WF Frame}

\subsection{Presentation of the Physical Model PM of the Two Swimmers}

The correct paths or routes in the PM for two boats $B_{1}$ and $B_{2}$ was described by us previously in [9] [10] [11]. The paths taken by the two boats now apply to the two swimmers Sw1 and Sw2, and are shown in Figure 2 and in Figure 3. These schematics represent the physical model of the two swimmers, which shall be analysed.

In Figure 2 and in Figure 3, the adopted reference frame is $x_{1} O_{1} y_{1}$, being attached to water, designated as water frame WF, with the axis $O_{1} x_{1}$ aimed reversely to water speed $v$. Here, point $O_{1}$ was taken as the point coinciding with the block-start (BS) right corner, and initial time $t=0$ is the moment when the race starts.

In this $\mathrm{PM}$, the water flows with speed $v$ towards the left side of observer $\mathrm{O}_{1}$ in Figure 2.

But in the WF selected here (in Figure 2 and in Figure 3), the block-start BS

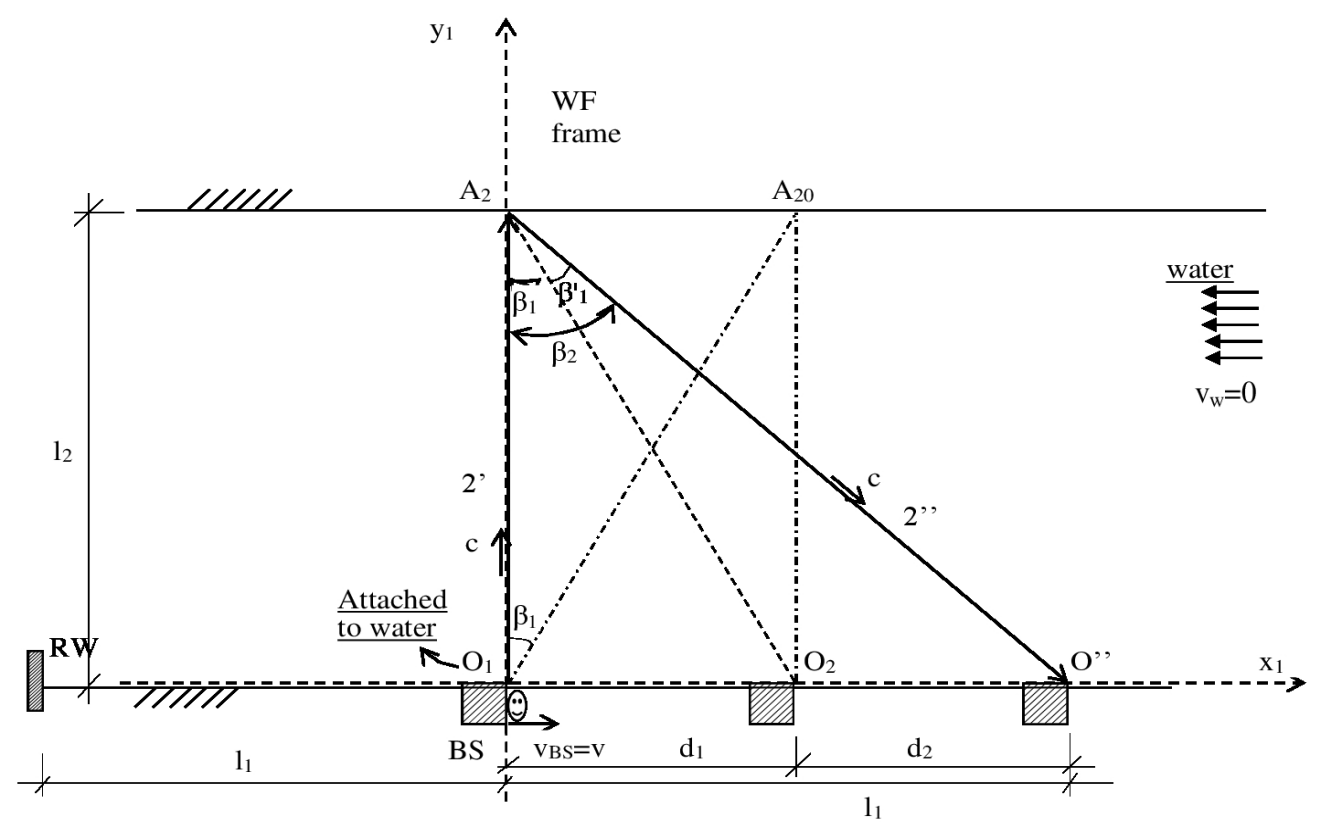

Figure 2. The PM model PM with two swimmers Sw1 and Sw2 in WF frame. The case of Sw2 in crosswise path $2^{\prime}+2$ " as right triangle. 


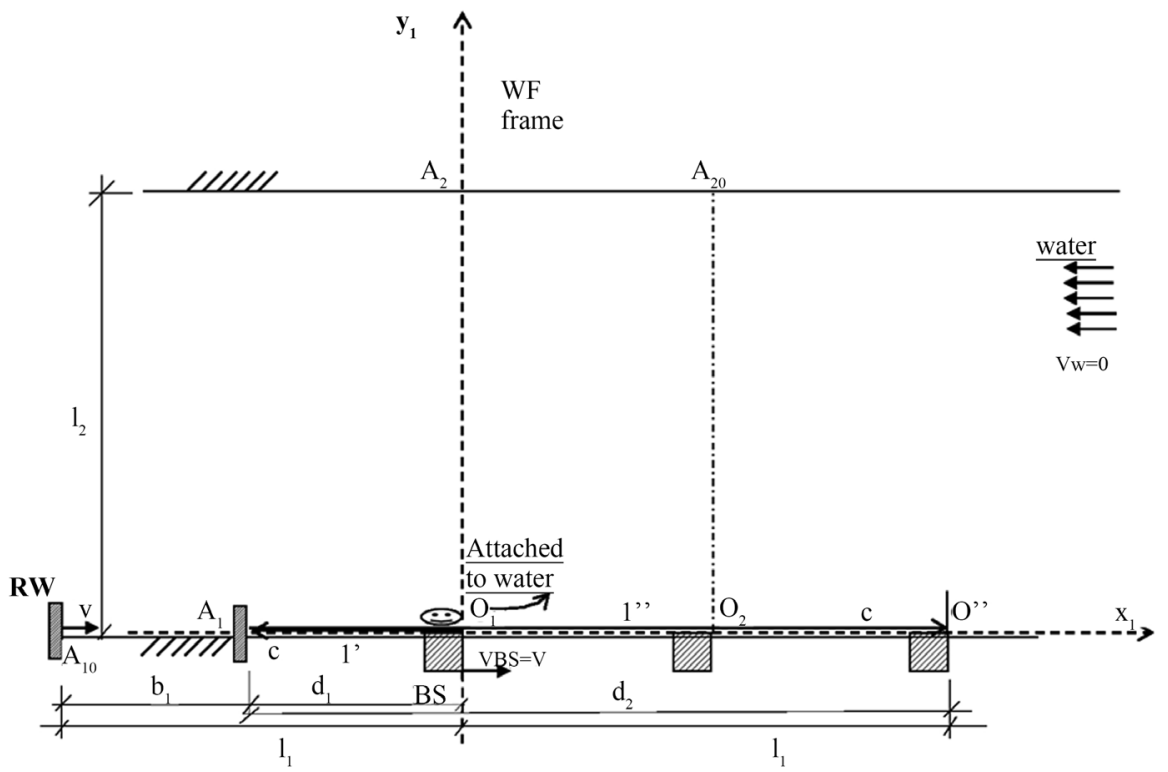

Figure 3. The model PM with two swimmers Sw1 and SW2, in WF frame. The case of Sw1 in longitudinal path 1' +1 '.

and the two river banks RB1 and RB2 will shift to the right side of the drawing with relative speed $v$ while the water remains still.

\subsection{Analysis of Model PM in Case of Crosswise Direction}

In $x_{1} O_{1} y_{1}$ frame at the time $t=0$, the swimmer Sw2 will leave from point $O_{1}$ in the direction $y_{1}$, which is perpendicular to the BS (and to the RB1), and it shall run his initial path 2' between the point $O_{1}$ and the point $A_{2}$ located oppositely on the opposed bank RB2 located at the orthogonal distance $I_{2}$ (Figure 2).

The time needed to cover path 2' $=O_{1} A_{2}$ shall be $t_{2}^{\prime}$, corresponding to running the path 2' by the swimmer Sw2 with his own speed $c$ against still water. Note that point $A_{2}$ does not coincide with point $A_{20}$ that corresponded to time $t$ $=0$ with the point on the RB2. This point $A_{20}$ is directly opposite to $O_{1}$ at that time $t=0$, because the opposite bank shifted to right with the speed $v$ in this timeframe $t_{2}^{\prime}$ on a distance $d_{1}$ (Figure 2).

At the same time, when Sw2 reaches point $A_{2}$, he can observe that the BS shifted with distance $d_{1}$ to the point $O_{2}$ (the point which is orthogonally opposite to point $A_{20}$ ) (Figure 2), and that BS continues to shift in the same direction $X_{1}$ with speed $v$. Therefore, in order for Sw2 to reach the exact meeting with BS at the return to point $O^{\prime \prime}$, Sw2 will have to also swim with speed $c$ to the estimated point of meeting $O^{\prime \prime}$ located at distance $d_{2} \approx d_{1}$ but still slightly longer than $d_{1}$ since time $t_{2}$ "is for return path 2" that will be sloped; path 2" is thus longer than 2' path (orthogonally on 1' path in the WF, see Figure 2).

Note that this correct and fair-play route 2' - 2" of swimmer Sw2 in the WF has the shape of a right triangle $O_{1} A_{2} O^{\prime \prime}$ in Figure 2 and not of a isosceles triangle as considered by authors from Sec. 2 and by Michelson. 
Now we will determine firstly these two times, $t_{2}^{\prime}$ and $t_{2}^{\prime \prime}$. As a reminder, first calculus concerning the two times, $t_{2}^{\prime}$ and $t_{2}^{\prime \prime}$, was performed by us in [9] [10] [11]. In those works, $t_{2}^{\prime}$ and $t_{2}^{\prime \prime}$ are the times spent by the light beams traversing their paths in the ME, and performed in two distinct sections, with separate meanings.

In order to better understand the final result of our calculus, i.e., $t_{2}=t_{1}$, we will resume here the calculus elaborated in [9] [10] [11] for time $t_{2}$ performed in the transversal path, and of time $t_{1}$ performed in the longitudinal path by the two swimmers Sw2 and Sw1 in the corresponding PM from Figure 2 and Figure 3, in WF frame.

We will improve here, some of our previous calculi, importing the two dedicated paragraphs and equations from [11] into the model PM from Figure 2 and Figure 3.

Also the size of small fonts of indices from some formulae from [11], was improved here, compared with [11].

We first determine the two times, $t_{2}^{\prime}$ and $t_{2}^{\prime \prime}$ with the geometrical elements of PM in Figure 2, from which it results that:

$$
\begin{gathered}
t_{2}^{\prime}=\frac{l_{2}}{c}=\frac{O_{1} A_{2}}{c} ; \quad t_{2}^{\prime \prime}=\frac{A_{2} O^{\prime \prime}}{c} \\
t_{2}=t_{2}^{\prime}+t_{2}^{\prime \prime} \quad O_{1} O^{\prime \prime}=v t_{2}
\end{gathered}
$$

From the right triangle $O_{1} A_{2} O^{\prime \prime}$ of Figure 2 it results that:

$$
\left(O_{1} A_{2}\right)^{2}+\left(O_{1} O^{\prime \prime}\right)^{2}=\left(A_{2} O^{\prime \prime}\right)^{2}
$$

Introducing (1) and (2) in (3) we obtain:

$$
\left(t_{2}^{\prime} c\right)^{2}+\left(v t_{2}\right)^{2}=\left(t_{2}^{\prime \prime} c\right)^{2}
$$

Replacing $t_{2}$ from (2) into (4) we obtain:

$$
\left(t_{2}^{\prime} c\right)^{2}+\left(v\left(t_{2}^{\prime}+t_{2}^{\prime \prime}\right)\right)^{2}=\left(t_{2}^{\prime \prime} c\right)^{2}
$$

After making the calculations between the brackets and regrouping the terms we obtain:

$$
\left(t_{2}^{\prime \prime}\right)^{2}\left(c^{2}-v^{2}\right)-2 v^{2} t_{2}^{\prime} t_{2}^{\prime \prime}-\left(t_{2}^{\prime}\right)^{2}\left(c^{2}+v^{2}\right)=0
$$

By solving the second-degree equation of (6) we obtain the result:

$$
\begin{aligned}
t_{2}^{\prime \prime} & =\frac{2 v^{2} t_{2}^{\prime}+\sqrt{4 v^{4}\left(t_{2}^{\prime}\right)^{2}+4\left(c^{2}+v^{2}\right)\left(c^{2}-v^{2}\right)\left(t_{2}^{\prime}\right)^{2}}}{2\left(c^{2}-v^{2}\right)} \\
& =\frac{v^{2} t_{2}^{\prime}+t_{2}^{\prime} \sqrt{v^{4}+\left(c^{2}+v^{2}\right)\left(c^{2}-v^{2}\right)}}{c^{2}-v^{2}}
\end{aligned}
$$

Due to the solution $t_{2}^{\prime \prime}=0$ obtained for sign (-) in front of the root sign of (7) would be pointless, we have chosen the sign $(+)$ in front of the root sign from Equation (7).

By introducing (1) and (7) in (2) we obtain: 


$$
\begin{aligned}
t_{2} & =\frac{l_{2}}{c}+\frac{\frac{v^{2} l_{2}}{c}+\frac{l_{2}}{c} \sqrt{v^{4}+c^{4}-v^{4}}}{c^{2}-v^{2}} \\
& =\frac{l_{2}}{c}+\frac{\frac{v^{2} l_{2}}{c}+\frac{c^{2} l_{2}}{c}}{c^{2}-v^{2}} \\
& =\frac{l_{2} c^{2}-l_{2} v^{2}+v^{2} l_{2}+c^{2} l_{2}}{c\left(c^{2}-v^{2}\right)}
\end{aligned}
$$

And from (8) we finally obtain for the time $t_{2}$ of the crosswise route 2' -2 ":

$$
t_{2}=\frac{2 l_{2} c^{2}}{c\left(c^{2}-v^{2}\right)}=\frac{2 l_{2}}{c} \frac{1}{1-v^{2} / c^{2}}=\frac{2 l_{2}}{c} \frac{1}{\alpha^{2}} \text { with } \alpha=\sqrt{1-v^{2} / c^{2}}
$$

\subsection{Analysis of Model PM in Case of Longitudinal Direction}

For the longitudinal direction, the path run by Sw1 is shown in Figure 3. It must also be specified that in Figure 3, the selected reference frame $x_{1} O_{1} y_{1}$, represents the water frame WF which is attached to water, and the axis $O_{1} X_{1}$ is aimed opposite to water speed $v$. Here we have taken the point $O_{1}$ of the initial moment $t=0$ of the race start, coinciding with the same right corner of the block start BS.

In Figure 3 at time $t=0$, swimmer Sw1 will leave from point $O_{1}$ in the direction reversed to $x_{1}$ and parallel to block-start BS (and to the RB1). His swim time is $t_{1}^{\prime}$ and his initial path $1^{\prime}$ is situated between the point $O_{1}$ and the point $A_{1}$, which is located in the already shifted position of the return wall RW (Figure 3 ). We note RW was initially (at $t=0$ ) situated on point $A_{10}$ of the same bank RB1 at a distance $l_{1}$.

The movement distance $b_{1}$ of RW in time $t_{1}^{\prime}$ and the movement distance $d_{1}$ of Sw1 in the same timeframe $0,1,2, \ldots t_{1}^{\prime}$ shall be (Figure 3):

$$
b_{1}=v t_{1}^{\prime} ; d_{1}=l_{1}-b_{1}=l_{1}-v t_{1}^{\prime}
$$

The time covered by Sw1 when going out and the covered distance shall be from (10):

$$
t_{1}^{\prime}=\frac{d_{1}}{c} ; d_{1}=l_{1}-v \frac{d_{1}}{c}
$$

From (11), after grouping the terms, it results for $d_{1}$ :

$$
d_{1}\left(1+\frac{v}{c}\right)-l_{1}=0 ; \rightarrow d_{1}=\frac{l_{1}}{1+v / c}
$$

And the time $t_{1}^{\prime}$ corresponding to the path $d_{1}$ run with speed $c$ shall be:

$$
t_{1}^{\prime}=\frac{d_{1}}{c}=\frac{l_{1}}{c+v}
$$

For the return path $t_{2}^{\prime \prime}$ of Sw1, the movement distance $b_{2}$ of RW and the movement distance $\mathrm{d}_{2}$ of Sw1 shall become (Figure 3):

$$
b_{2}=v t_{1}^{\prime \prime} ; \quad d_{2}=l_{1}+b_{2}=l_{1}+v t_{1}^{\prime \prime}
$$

The time $t_{2}^{\prime \prime}$ covered by Sw1 when returning and the covered distance $d_{2}$, 
shall be from (14):

$$
t_{1}^{\prime \prime}=\frac{d_{2}}{c} ; d_{2}=l_{1}+v \frac{d_{2}}{c} ;
$$

From (15), after grouping the terms, it results $d_{2}$ :

$$
d_{2}\left(1-\frac{v}{c}\right)+l_{1}=0 ; \rightarrow d_{2}=\frac{l_{1}}{1-v / c}
$$

And time $t_{2}^{\prime \prime}$ corresponding to returning route $d_{2}$ covered with speed $c$ becomes:

$$
t_{1}^{\prime \prime}=\frac{d_{2}}{c}=\frac{l_{1}}{c-v}
$$

Therefore, total time $t_{1}$ shall be from (13) and (17):

$$
t_{1}=t_{1}^{\prime}+t_{1}^{\prime \prime}=\frac{l_{1}}{c+v}+\frac{l_{1}}{c-v}=\frac{2 l_{1} c}{c^{2}-v^{2}}=\frac{2 l_{1}}{c} \frac{1}{\alpha^{2}}
$$

Note that in (18) the same result was obtained as in (9) except for lengths $I_{1}$ and $l_{2}$. Hence, in case of length equality $l_{1}=l_{2}$, it results from (9) and from (18):

$$
t_{2}=t_{1} ; \text { and } \Delta t=t_{2}-t_{1}=0
$$

Therefore, swimmers Sw1 and Sw2 shall arrive at the same time if at their departure the fair-play of orthogonality for their starting direction will be also respected for Sw2. Their race would thus end in a tie as we demonstrated above. No winner yields this race, just hypothetical, because there are no data or proofs that such a race would have been practically performed and Sw2 was the winner. But indeed a real such a contest would be clearer for the disputed result, analysed above.

Moreover, any intermediary starting direction of 1' path with starting angle $\alpha$ $\neq 0$ of Sw1 may be considered as also obtainable from the overlapping of the two cases of $0^{\circ}$ and $90^{\circ}$ analysed above, but with the results of times $t$ applied in percentages appropriate for $\alpha$. And such one results that the obtained equality of the two times, $t_{2}=t_{1}$ shall be obtained for any pair of paths in which the paths 1 ' and $2^{\prime}$ will be aimed at $90^{\circ}$ between them, independently of the starting angle $\alpha$ for Sw1.

\section{Correct Reanalysis of the Physical Model PM in BF Frame}

\subsection{Presentation of the Physical Model PM of the Two Swimmers in BF Frame}

The routes of the two boats $\mathrm{B} 1, \mathrm{~B} 2$ in the $\mathrm{BF}$ reference frame within the PM, that was followed correctly and in the fair-play, according to the appropriate rules described in Sec. 1, has been already detailed previously in [9] [10] [11].

The paths for the two boats are adapted for the swimmers Sw1 and Sw2 and are shown in Figure 4. Here, the reference frame is $x O y$, which is attached to the block-start, being a BF frame, with the axis $O x$ aimed oppositely to water speed $v$. 


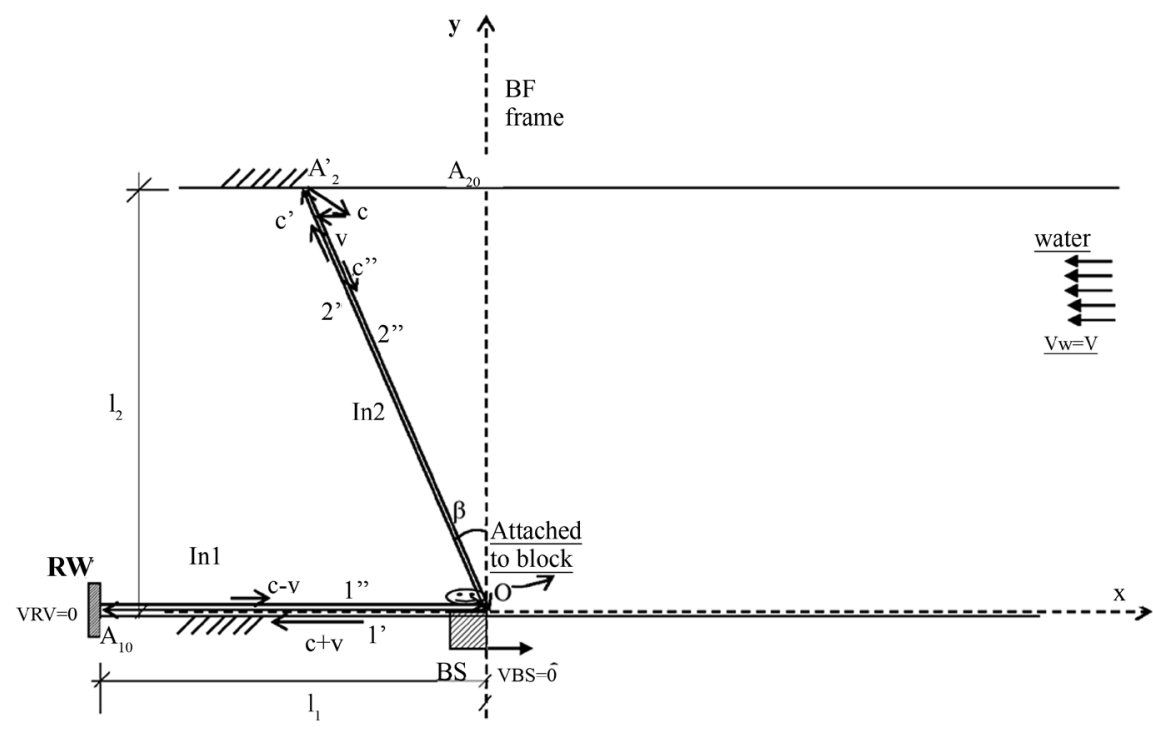

Figure 4. The model PM with two swimmers Sw1 and Sw2 in BF frame. The case of longitudinal path 1' +1 " and of transversal path 2 ' +2 " inclined with $\beta$ double line.

Additionally, point $O$ at initial time $t=0$ coincides with the block-start BS right corner. In this model, we will consider that the water flows with the speed $V$ pointed left relative to observer $O$ from Figure 4, while the block-start BS, the two river banks RB1 and RB2, and the return walls RW shall remain still for the observer from the $\mathrm{BF}$ frame.

\subsection{Analysis of Model PM in Case of Transversal Direction}

In Figure 4, at the time $t=0$, swimmer Sw2 leaves from point $O$ with his speed $c$ in the direction $y$, perpendicular to the block-start BS (and to the RB1 bank).

As Sw2 swims in the river, he will be carried over by the water with speed $v$. Therefore, the speed of Sw2 swimmer against block-start BS shall be $c$ 'inclined with an angle $\beta$, depending also on the velocity triangle [9] [10] [11]. Sw2 shall cover his initial path 2' between point $O$ and point $\mathrm{A}_{2}$, situated on the opposite bank RB2 (Figure 4) but located at a distance $d_{1}$ given by angle $\beta$ in BF, $d_{1}=$ $O A_{2}^{\prime}$. Swimmer Sw2 shall cover this distance $d_{1}=I_{2} v / c$ within the timeframe $t_{2}^{\prime}$.

Note that point $A_{2}^{\prime}$ does not coincide with point $A_{20}$ (Figure 4), which corresponded to initial time $t=0$ on a point of the RB2 bank, that was directly opposite to point $O$ at that time.

As Sw2 returns from $A_{2}^{\prime}$ to starting point $O$ (Figure 4), he swims with his own speed $c$, but angled against axis $O y$, at an angle $\varepsilon$, depending on the velocity triangle [9] [10] [11], as such that under the influence of water speed $v$, the resultant speed $c$ " is aimed toward point $O$.

Sw2 is able to see the point $O$ right from the moment of his return from point $A_{2}^{\prime}$ (Figure 4), without breaking any fair-play rule. Hence, Sw2 shall return to point $O$ after the timeframe $t_{2}^{\prime \prime}$. The complete transversal/crosswise path in BF frame takes the shape of an incline with angle $\beta$ double line (Figure 4 ). 
Because the model PM as physical phenomenon involved, or even because the real contest is unique in reality, then in any selected reference frame WF or BF, the total time $t_{2}=t_{2}^{\prime}+t_{2}^{\prime \prime}$ of running the above path 2' $-2^{\prime \prime}$, must be equal to the calculated time $t_{2}$ of Equation (9) and it does not require new calculations here.

It is logical that such calculations performed for the path 2' - 2" from Figure 4 shall yield for $t_{2}$ the result of Equation (9). The timeframes $t_{2}^{\prime}$ and $t_{2}^{\prime \prime}$ of the two frames WF and BF which must be equal between them.

\subsection{Analysis of Model PM in Case of the Longitudinal Direction}

For the longitudinal direction, the path 1' +1 " covered by Sw1 is also shown in Figure 4. Here, we have taken point $O$ of the initial time $t=0$ to be the start of the race and have it coincide with the same block-start BS right corner.

When $t=0$, Sw1 shall leave from point $O$ toward a direction opposite to $\mathrm{O} x$ and parallel to the block-start (and to RB1). He shall cover in $t_{2}^{\prime}$ time his initial path 1' between point $O$ and point $A_{10}$, which is also located in the initial position of the return wall RW in BF (Figure 4).

For the return path 1", Sw1 shall start from point $A_{10}$ in the direction of $O$ where he shall arrive after $t_{2}^{\prime \prime}$ time on position $O$, which is still the initial one in BF (Figure 4). His return path to $O$ takes a total timeframe $t_{1}=t_{2}^{\prime}+t_{2}^{\prime \prime}$.

However, the rule of times $t_{2}$ discussed at the end of Sec. 6.2 shall also apply to this go-return longitudinal path. This is regarding the equivalence also of the times $t_{1}$ covered in the two reference frames BF and WF. Therefore, the total time $t_{1}=t_{2}^{\prime}+t_{2}^{\prime \prime}$ for running the path 1' -1 "' (Figure 4) must be equal to the calculated time $t_{1}$ in WF from Equation (18). It does not require new time calculations in BF. Also the partial timeframes, $t_{2}^{\prime}$ and $t_{2}^{\prime \prime}$, of the two cases WF and $\mathrm{BF}$, must to be equal between them. Neither one of them require a new calculation.

\section{Conclusions and Consequences}

From the above-presented situations of the physical model (PM), the path taken by swimmer Sw2 in the crosswise direction of the river flow with respect to the water reference frame WF must take the shape of a right triangle. This disagrees with interpretations from many other authors and from Michelson himself, who considered the isosceles triangle to be the correct path (probably also in WF). The context is in the analytical interpretation of the Michelson interferometeric experiment (ME).

When considering the right triangle of the path covered by Sw2 in WF, the two times of swimmers Sw1 and Sw2 shall be equal, i.e., $t_{1}=t_{2}$ and their race ends in a tie.

In the BF frame, the transversal path takes the shape of an inclined double line, with an angle $\beta$, but the times $t_{1}$ and $t_{2}$ must remain the same as in the WF frame. 
As a consequence, utilising the isosceles triangle for the transversal path from the PM in the WF frame, results in an erroneous analysis.

Clearly, this result should also be applied to other similar experiments, as well to the analysis of ME experiment. Therefore, the presence of the ether remains a possibility even after the ME experiment correct interpreted as above, and the ether should not be removed from physics, as Einstein did based on his interpretation of the ME.

Based on our above results it is justified in physics, to renounce in future at SRT, and instead to reintroduce a modern real ether in physics, as we already done in two published papers [12] [13], including a new gravitation theory. And so in presence of the ether, many unexplained phenomena, ranging from quanta entanglement in microcosm, the atmospheric electricity at earth scale, and so called dark matter in astrophysics, and others such phenomena as so called black holes, will receive their physical explanation.

\section{Conflicts of Interest}

The authors declare no conflicts of interest regarding the publication of this paper.

\section{References}

[1] Freund, J. (2008) Special Relativity for Beginners. A Textbook for Undergraduates. Word Scientific Publishing Co Ltd., 6. https://www.worldscientific.com/doi/suppl/10.1142/6601/suppl_file/6601_chap01.p df

[2] Harrison, D.M. (2007) The Special Theory of Relativity. 2. https://faraday.physics.utoronto.ca/GeneralInterest/Harrison/SpecRel/SpecRel.pdf

[3] Glesson, A. (2007) Class Notes. Introduction to Relativity-Physics 375R, 20. https://web2.ph.utexas.edu/ gleeson/RelativityNotesChapter1.pdf

[4] Fowler, M. (2008) Notes on Special Relativity. CreateSpace Independent Publishing Platform. 14. http://galileo.phys.virginia.edu/classes/252/SpecRelNotes.pdf

[5] Jafe, B. (1967) Albert Michelson and Light Speed (Albert Michelson si viteza luminii. In Romanian) Ed. Stiintifica, Bucuresti, 52-53.

[6] Gamow, G. (1958) One, Two, Three, ...Infinite. (Unu, doi, trei, ...infinit. In Romanian). Ed. Tineretului, Bucuresti, 101-103.

[7] Ciobotaru, D., Angelescu, T., Munteanu, I., Melnic, M. and Gall, M. (1997) Physics. 12th Grade Textbook, (Fizica. Manual cl. XII-a. In Romanian). Editura Didactica si Pedagogica, Bucuresti, 7-8.

[8] Le, T.D. (2005) The Special Theory of Relativity. 23. http://thehuuvandan.org/relativity.html

[9] Has, I. (2000) The Analysis of an Alternative Light Path in Michelson's Experiment. Romanian Reports in Physics, 52, 775-789.

[10] Has, I., Miclaus, S. and Has, A. (2010) An Alternative Light Path Analysis in Michelson's Experiment. Physics Essays, 23, 248-257. https://doi.org/10.4006/1.3357572

[11] Has, I. Miclaus, S. and Has, A. (2014) A Reanalysis of the Theory of Interferometer 
Experiment Demonstrating that Michelson's Analysis Contains an Error, Including the Boat Model Analysis, So Readmitting the Ether Presence. Optics, 3, 24-32.

http://www.sciencepublishinggroup.com/j/optics https://doi.org/10.11648/j.optics.20140304.11

[12] Has, I., Miclaus, S. and Has, A. (2015) A Theoretical Confirmation of the Gravitation New Origin Having a Dipolar Electrical Nature with Coulomb Law Corrected. American Journal of Modern Physics, 4, 97-108.

http://www.sciencepublishinggroup.com/j/ajmp

https://doi.org/10.11648/j.ajmp.20150403.11

[13] Has, I., Miclaus, S. and Has, A. (2016) An Initial Model of Ether Describing Electromagnetic Phenomena, Including Gravity. Physics Essays, 30, 45-56.

https://doi.org/10.4006/0836-1398-30.1.45 